starch degradable to maltose, which forms the bulk of the granule, amylocellulose or amylopectin, and a small proportion of carbohydrate, possibly crystalline, soluble in cold water. It is probable that these are present in different proportions in the various starches, and so give rise to such differences as Reichert has observed.

Some valuable observations on the characters of hard and tender barleys, published $\mathrm{H}$. C. A. Vine in the Journal of the Institute of Brewing, may be mentioned in this connection. A barley corn may contain starch granules of all sizes, the variation being due to the conditions under which it develops. Malnutrition of barley leads to a high ratio of small starch granules which are more resistant to enzyme action, to moisture, and to heat than the normal mature granules. Those granules which have the more favourable position in the enclosing cell are able to appropriate a large proportion of the nutriment supplied by the protoplasm, and so become normal large starch granules, each consisting of many layers containing much granulose, tender and readily acted upon.

Similar observations were made by the writer some years back when it was pointed out that there is considerable variation in the proportion of large to small granules in wheat starch. Those types of flour which are the best for certain purposes contain the greatest proportion of large granules, the property being quite characteristic.

Hence it would seem that, over and above species variation, differences due to environment and nurture may appear in the starches, and it is possible that the further study of such a substance as starch may provide material for the solution of many vexed problems.

In addition to the detailed account of the tests applied to each starch, which are recorded also in the form of a curve which is shown to be characteristic for each individual, Prof. Reichert includes in his book a beautiful series of photomicrographs taken in ordinary and polarised light. These enhance very materially the value of the work, although they must have increased greatly the cost of publication. The author has further been at pains to summarise at some length previous work on starch, both on the chemical and on the botanical side. His account is a valuable one if only as showing how much uncertainty exists at present in the knowledge of starch and its transformations.

E. F. A

\section{TRANSPIRATION IN PLANTS.}

TWO paper by Sir Francis Darwin (Proceedings of the Royal Society, B, vol. lxxxvii.) mark an important advance in the study of the process of transpiration in plants. Hitherto, although transpiration is perhaps more directly under the rule of external physical conditions than any other physiological function of plants, there has been no complete experimental demonstration of the relation between the loss of water-vapour from leaves and the relative humidity of the air or of the effect on transpiration of variation in the illumination to which the leaf is subjected. These lacunæ are due to the fact that transpiration depends largely on the opening and closing of the stomata, the aperture of which varies in area with varying external conditions. To eliminate from the problem the varying stomatal aperture, the author blocks the stomata by smearing the leaf with cocoafat or vaseline, and then makes incisions which place the intercellular spaces in communication with the atmosphere; by measuring the thickness of the leaf and making incisions of a certain total length, the area thus exposed is made to correspond with the area of the stomatal apertures under ordinary conditions By using this ingenious method, the author finds that the line joining the abscissce representing the transpiration for different degrees of atmospheric humidity is practically straight, but that the transpiration begins at about 5 per cent. above saturation, and from calculation of the vapour pressures at saturation and this degree of supersaturation, it appears that the internal temperature of the leaf which can distil off vapour in saturated air is about $\mathrm{I}^{\circ} \mathrm{C}$. above that of the air, this increased temperature being attributable to respiration.

The second paper gives the results obtained by applying this method to the investigation of the effect of light on transpiration. In April the transpiration of ivy was the same in diffused daylight and in dark ness, while a month later the transpiration in light was double that in darkness, but the average ratio for transpiration in light and darkness was I35: 100, though between May I4 and June I6 the laurel gave an average $150:$ roo. The cause of the increased reaction to illumination in early summer as compared with spring is not completely explained, the author having no evidence as to whether the increased permeability of the leaves to water is a periodic effect, or connected with the age of the leaf, or with the brightness of the summer sky, as compared with illumination earlier in the year

\section{THE AMERICAN PHILOSOPHICAL SOCIETY.}

THE annual general meeting of the American Philosophical Society was held in Philadelphia on April 23-25 inclusive, when numerous papers embodying the results of original researches were read. It is possible here to refer only to the more important and to those of wide scientific interest.

The president, Dr. William W. Keen, was in the chair at the opening meeting, and among the papers presented was one on the segregation of "unit characters" in the zygote of CEnothera with twin and triplet hybrids in the first generation, by Prof. G. F. Atkinson, Cornell University. The segregation of several distinct hybrid types in the first generation of a cross between two species is a rare phenomenon. In Prof. Atkinson's experimental studies, the two parents are Enothera nutans and $E$. pycnocarpa, wild species of the evening primrose in the vicinity of Ithaca, N.Y. They differ by more than thirty easily recognisable contrast pairs of "unit characters," or, in terms of the "presence and absence" hypothesis, there are more than sixty "factors" or recognisable characters which meet in the fertilised egg of the cross between the two parents. These characters relate to the habit and colour of the adults, features of the rosettes, foliage, and inflorescence. When pycnocarpa is the mother, two distinct hybrid types are segregated in the first generation, and have been brought to maturity. These are "twin hybrids." When nutans is the mother, the same twin hybrids appear, and, in addition, a triplet which at present is in the rosette stage.

The analysis of the hybrids shows a distinct linking or association of certain characters. Examples of this linking of characters are as follows:-First, habit characters; secondly, colour characters ; thirdly, petal characters; fourthly, broadness and toothedness of rosette leaves; fifthly, narrowness and cutness of rosette leaves; sixthly, crinkledness, convexity, and red-veinedness of rosette leaves; seventhly, plainness, furrowedness, and white-veinedness of rosette leaves.

The following hypotheses are considered:-(I) De 
Vries's hypothesis of twin hybrids from mutating specses; (11) theory of a differential division in the zygote; and (iii) the reaction theory.

A paper on the vegetation of the Sargasso Sea was contributed by Prof. W. G. Farlow, of Harvard University. The Sargasso Sea is characterised by the scattered masses of gulf weed which float on the suriace of the ocean in patches from 50 to roo $\mathrm{ft}$. in diameter. Some consider that the gulf weed, Sargassum bacciferum, is merely a mass of sterile branches of some species of Sargassum, which grows attached in the region of the West Indies, and truits. Others believe that in its present floating form it is a distinct species which has lost the power of fruiting and increases only by offshoots. In recent years the species of Sargassum growing in different parts of the West Indies have been studied, and a comparison with the floating gulf weed shows that mixed with it are found fragments of at least two species known to grow in the West Indies. In only one instance has there been found mixed with the gulf weed a seaweed which must have come, not from the American coast, but from Africa or southern Europe. There is reason to think that the gulf weed is derived from some Sargassum growing in the West Indies, fragments of which are carried by the Gulf Stream to the Sargasso Sea.

On April 24 a paper on phase changes produced by high pressures was read by Mr. P. W. Bridgman, of Harvard University. Pressures as high as 30,000 or $40,000 \mathrm{kgm}$. per sq. cm. were employed. Examination of the melting of a number of liquids over a wide pressure range has shown that the theories hitherto proposed do not hold at high pressures. So far as can be judged the melting curve continues to rise indefinitely, so that a liquid may be frozen by the application of sufficient pressure, no matter how high the temperature. A number of results are also obtained for the reversible transition from one crystalline form to another. Several new solid forms have been obtained; of particular interest are the new forms of ice which are denser than water. In addition to these changes, which are completely reversible, one example has been found of an irreversible reaction produced by high pressure; yellow phosphorus may be changed by $12,000 \mathrm{kgm}$. and $200^{\circ}$ to a modification in appearance like graphite, which is $\mathrm{I}_{5}$ per cent. more dense than the densest red phosphorus.

Prof. R. A. Millikan, of the University of Chicago, read a paper on some new tests of quantum theory and a direct determination of $h$. It has been known for twenty-five years that when light of sufficiently short wave-length falls upon a metal, it has the power of ejecting electrons from that metal. It has been known for seven years that the kinetic energy possessed by the electrons thus ejected is larger the higher the frequency of the light which ejects them. Whether or not the energy of ejection is directly proportionate to frequency has been a matter of some uncertainty. Prof. Millikan's work furnishes proof that there is exact proportionality between the energy of the ejected electrons and the frequency of the light which ejects them, and that the factor of proportionality between the energy of the ejected electrons and the frequency of the incident light is the same quantity as the fundamental constant which appears in Planck's theory of the discontinuous or explosive character of all radiant energy of the electromagnetic type. This constant is known as Planck's $h$, and its value is directly determined with an error which does not exceed I per cent.

Dr. Charles F, Brush, of Cleveland, discussed "A Kinetic Theory of Gravitation : (I) Gravitation is Due to Intrinsic Energy of the Æther; (2) Transmission of
Gravitation cannot be Instantaneous." He employs illustrations to show that the energy acquired by falling bodies has some external source, and that it must be æetherial energy or energy of space; and he holds that the term "potential energy of position," as applied to a system of gravitating bodies, implies the energyendowed æther as a necessary part of the system. As a corollary, he explains how bodies falling toward each other by reason of their mutual attraction, and thus accelerating - that is to say, absorbing energy from the æther-cannot rigidly obey Newton's law of inverse squares of distance. In the second division of the paper the premises from which Laplace drew his famous conclusion that gravitation is transmitted with infinite, or virtually infinite, velocity, are described, a dogma which, said Dr. Brush, "for more than a century has blocked the path of fruitful thought on the physics of gravitation." It is concluded that, even if the velocity of transmission is no greater than that of light, the moon's mean motion will be retarded a very few seconds of arc only, in a century; and the retardation will be correspondingly less if the velocity is greater than that of light. This retardation, of course, adds to the unexplained acceleration, if any, of the moon's motion; but the author further hopes that this retardation, plus the outstanding acceleration, will be explained by a particular deviation from Newton's law described.

Prof. W. Duane, of Harvard University, presented a contribution on highly radio-active solutions. The advantage in using these solutions in studying the effects produced on tissues is that after injection the radio-active substances come into intimate contact with the tissues, and thus the full power of the alpha rays is utilised. If a solution of radium itself is injected, the process is not only costly, but dangerous, on account of the long life of the radium. The solutions do not have these objections, for the radium is not wasted in producing the solutions, and the activity lasts for only a short time. If the injection is made subcutaneously, a large fraction of the activity remains in the neighbourhood of the point of injection, and the rest is carried off in the lymph and blood streams. The rapidity with which the activity gets into circulation is astonishing. A drop of blood taken from another part of the body only a few seconds after the injection is more radio-active than carnotite or pitchblende orcs. It would seem that this might prove to be a delicate method of studying the flow of fluid through the tissues. On making tests by means of the gamma rays an hour or an hour and a half after the injection it was found that there was very little activity in the brain and lungs, but that there was a tendency for the substances to deposit out in the liver, spleen, and kidneys.

"The Relations of Isostasy to a Zone of Weakness -the Asthenosphere," was the subject of a paper by Prof. J. Barrell, of Yale University. The mass of every mountain tends to deflect the plumb-line slightly, so that the measured latitude and longitude of any locality will differ as it is determined by triangulation or by astronomic determination of the point in which the observed vertical pierces the celestial sphere. But Hayford has shown that the deflections of the vertical are actually only one-tenth of the deflections calculated as due to the terrestrial relief. This is a quantative test of the degree of isostasy. Dynamically it implies a state of flotation of the crust upon the inner earth analogous to the flotation of an iceberg in the ocean. Yet the earth as a whole is known to be as rigid as steel; the nature of earthquake vibrations transmitted through the earth shows it to be solid throughout and more incompressible and rigid at great depths than near the surface. How, then, shall the geodetic 
evidence poincing toward a general flotation of the crust near to equilibrium be reconciled with this other evidence of great rigidity and strength? It has been supposed that a mobile zone may explain the apparent contradiction, but the necessity of postulating such a zone becomes greater as the accumulated evidence of weakness on one hand, of strength on the other, diverges more and more. By means of a study of the areas of the surface loads and their degree of departure from isostatic equilibrium this zone is located far deeper than other estimates have placed it, the level of minimum strength being thought to lie as much as 150 to 200 miles deep. The maximum strength is probably at a depth of ten to twenty miles, and falls off rapidly below.

"The Geologic and Biologic Results of a Study of the Tertiary Floras of South-eastern North America" were presented by Prof. E. W. Berry, of Johns Hopkins University. The results of many years of study of the rich Tertiary floras of south-eastern North America were announced for the first time. Their botanical relationships and their bearing on the evolution of types and upon geographical distribution were summarised. The studies have afforded for the first time fossil floras of fixed stratigraphic position for comparison with the floras of the Rocky Mountain province on the border between the Cretaceous and Tertiary, the age of which has caused much controversy. They also afford means for correlation with the type of section of the Paris basin.

During the afternoon of April 24 a portrait of the late Dr. S. P. Langley, who was a former vicepresident, was presented to the society by Dr. C. Adler on behalf of a number of members. On April 25 the following new members were elected as the result of balloting,-Mr. C. G. Abbot, Washington; Dr. J. W. Bright, Baltimore; Dr. B. M. Davis, Philadelphia; Dr. T. McCrae, Philadelphia; Dr. W. D. Matthew, New York; Dr. A. G. Mayer, Washington; Dr. S. J. Meltzer, New York; Dr. J. C. Merriam, Berkeley, Cal. ; Prof. R. A. Millikan, Chicago; Prof. W. A. Noyes, Urbana, I11.; Dr. Stewart Paton, Princeton; Dr. R. M. Pearce, jun., Philadelphia; Dr. P. C. Ricketts, Troy; Dr. Harold A. Wilson, F.R.S., Houston; Dr. F. E. Wright. Washington; Dr. Shibasaburo Kitasato, Tokyo; Prof. H. Kamerlingh Onnes, Leyden: and Dr. Vito Volterra, Rome.

At the concluding session of the meeting a medallion portrait of the late Sir Joseph D. Hooker was unveiled by Prof. W. G. Farlow, of Harvard University.

\section{X-RAYS AND CRYSTALLINE STRUCTURE: 1}

TWO years have gone by since Dr. Laue made his surprising discovery of the interference effects accompanying the passage of $\mathbf{X}$-rays through crystals. The pioneer experiment has opened the way for many others, and a very large amount of work, theoretical and practical, has now been done. As the preliminary exploration of the new country has proceeded our first estimate of its rescurces has grown continuously; we have learnt many things which help us to a better understanding of phenomena already familiar, and we have seen avenues of inquiry open out before us which as yet there has been little time to follow. The work is full of opportunities for exact quantitative measurement, where precision is sure to bring its due reward. There is enough work in sight to absorb the energies of many experimenters, and there is sure to be far more than we can see. When we consider the wideness of the new field, the quality and quantity of the work to be done in it, and the importance

1 Niscourse delivered at the Royal Institution on June 5 by Pror. IV. H. Bragg, F R.S.

NO. 2332, VOL. 93] of the issues, we are scarcely guilty of over-statement if we say that Laue's experiment has led to the development of a new science.

The experiment itself--to put it very briefly-constitutes a proof that X-rays consist of extremely short æther waves. In order to appreciate the value of this demonstration, we must bear in mind the presen. conditions of our knowledge of the laws of radiation in general. Let us consider very shortly how the whole matter stood when the new work was begun.

When X-rays were first discovered eighteen years ago it was soon pointed out that they might consist of electromagnetic disturbance of the æther analogous to those supposed to constitute light. It was true that the new rays seemed to be incapable of reflection, refraction, diffraction and interference which were familiar optical phenomena. But it was pointed out by Schuster (NATURE, January 23, 1896 ) that these defects could be explained as natural consequences of an extremely small wave-length. The positive evidence consisted mainly in the knowledge that the impact of the electrons on the antikathode of the X-ray bulb ought to be the occasion of electromagnetic waves of some sort, and in the discovery by Barkla that the X-rays could be polarised, which last is a property also of light.

As experimental evidence accumulated, a number of results were found which the electromagnetic theory was unable to explain, at least in a direct and simple manner. They were mainly concerned with the transference of energy from place to place. In some way or other the swiftly moving electron of the X-ray bulb transfers its energy to the X-ray, and the X-ray in its turn communicates approximately the same quantity of energy to the electron which originates from matter lying in the track of the $\mathrm{X}$-ray, and which is apparently the direct cause of all X-ray effects. Experiment seemed to indicate that X-ray energy travelled as a stream of separate entities or quanta, the energy of the quantum differing according to the quality of the X-ray. It looked at one time as if it might be the simplest plan to deny the identity in nature of X-rays and light, to describe the former as a corpuscular radiation, and the latter as a wave motion. Otherwise it seemed that the electromagnetic hypothesis would be torn to pieces in the effort to hold all the facts together.

But it appeared on a close examination of light phenomena also, though in much less obvious fashion, that the very same effects occurred which in X-rays were so difficult to explain from an orthodox point of view. In the end it became less difficult to deny the completeness of the orthodox theory, than the identity in nature of light and X-rays. Modern work on the distribution of energy in the spectrum, and the dependence of specific heat upon temperature have also led independently to the same point of view. It has been urged with great force by Planck, Einstein, and others that radiated energy is actually transferred in definite units or quanta, and not continuously: as if we had to conceive of atoms of energy as well as of atoms of matter. Let it be admitted at once that the quantum theory and the orthodox theory appear to stand in irreconcilable opposition. Each by itself correlates great series of facts; but they do not correlate the same series. In some way or other the greater theory must be found, of wlich each is a partial expression.

The new discovery does not solve our difficulty at once, but it does two very important things. In the first place, it shows that the $\mathrm{X}$-rays and light are identical in nature; in fact, it removes every difference except in respect to wave-length. The question as to the exact place where the difficulty lies is decided for us; we are set the task of discovering how a continuous wave motion, in a continuous 